Itinéraires Itinéraires

Littérature, textes, cultures

2014-1 | 2015

Textualités numériques

\title{
Ce qui s'écrit dans les univers numériques
}

Matières technolangagières et formes technodiscursives

\section{Marie-Anne Paveau}

\section{OpenEdition}

Journals

Édition électronique

URL : http://journals.openedition.org/itineraires/2313

DOI : $10.4000 /$ itineraires.2313

ISSN : 2427-920X

Éditeur

Pléiade

\section{Référence électronique}

Marie-Anne Paveau, "Ce qui s'écrit dans les univers numériques », Itinéraires [En ligne], 2014-1 | 2015, mis en ligne le 12 janvier 2015, consulté le 19 avril 2019. URL : http://journals.openedition.org/

itineraires/2313 ; DOI : 10.4000/itineraires.2313

Ce document a été généré automatiquement le 19 avril 2019

\section{(c) $(1) \odot$}

Itinéraires est mis à disposition selon les termes de la licence Creative Commons Attribution - Pas d'Utilisation Commerciale - Pas de Modification 4.0 International. 


\title{
Ce qui s'écrit dans les univers numériques
}

\author{
Matières technolangagières et formes technodiscursives
}

Marie-Anne Paveau

\section{Introduction}

1 L'objet posé ici est le discours natif en ligne ${ }^{1}$ abordé avec les outils de la théorie du discours ouverte sur la dimension cognitive du côté de la cognition distribuée, c'est-àdire externe, culturelle et sociale, et non internaliste et neuronale (Paveau 2012b, 2012c). S'y ajoutent ceux de l'analyse du discours numérique, fondée sur la notion de technologie discursive et sur le décentrage de l'objet habituel des linguistiques TDI (texte, discours, interactions) : travailler sur les univers discursifs numériques implique de dépasser une conception logocentrée (centrer l'analyse sur les énoncés et les seules matières langagières) pour élaborer une conception écologique qui observe dans leur entier les environnements des énoncés (Paveau 2009).

2 L'objectif de ce travail est de saisir les spécificités linguistiques de l'écriture en ligne dans ses environnements natifs, pour montrer comment la dimension technique configure les scripturalités. Il voudrait également permettre de montrer que, pour étudier les énoncés en ligne, la connaissance des spécificités des contextes numériques est nécessaire: Internet, et le Web en particulier, ne constituent pas de simples supports pour une production scripturale qui s'y transporterait, mais bien des environnements qui configurent structurellement les écritures de manière spécifique.

3 Après l'exposé du cadrage épistémologique et théorique de mon travail, je distinguerai trois catégories de scripturalité numérique (numérisée, numérique et numériquée), et j'interrogerai la manière dont l'énonciation, au fondement des linguistiques TDI, doit être repensée à la lumière des corpus de discours en ligne. 


\section{Un cadre postdualiste pour penser la textualité numérique}

4 J'appelle postdualistes les approches qui remettent en cause les grands binarismes fondateurs de la pensée occidentale, en particulier, pour ce qui m'intéresse ici, les oppositions esprit vs monde, langue vs monde et humain vs non-humain (Achard-Bayle et Paveau 2012). Le postdualisme pense l'humain comme articulé à ce qui n'est pas lui, et non plus dans une position exceptionnelle ou dominante au sein de l'univers (Paveau 2012c). C'est dans cette épistémè qu'il me semble possible de penser les écritures numériques comme intrinsèquement liées à leurs environnements natifs.

\subsection{L'intégration du non-humain}

5 La théorie des affordances proposée à la fin des années 1970 par le psychologue Gibson (1979), puis reprise par le designer Norman (1988), attribue aux objets des capacités cognitives et en particulier celle de fournir (to afford) aux utilisateurs des instructions pour leur usage. Sous cette approche, les outils technologiques soutenant les univers discursifs numériques (ordinateurs, programmes et applications) participent à l'élaboration du sens: discours, objets techniques et outils numériques doivent être abordés en système, dans une perspective écologique (Paveau 2013b).

6 La cognition sociale va dans le même sens puisqu'elle postule que les cadres et représentations qui guident nos actions, y compris la production de nos énoncés, sont externes. Ce domaine, qui propose à la fin des années 1980 une alternative à la cognition dite orthodoxe (internaliste), s'enrichit vite de plusieurs propositions (cognition située, cognition socialement partagée, cognition distribuée), autour des travaux de Suchman, Lave, Hutchins, Latour, Resnick, Schegloff entre autres. La cognition distribuée est celle qui fait le plus de place au non-humain, en particulier aux dispositifs techniques : dans son ouvrage Cognition in the Wild, Hutchins fait la synthèse de cette approche, qui saisit la manière dont les informations et les représentations se propagent dans les systèmes fonctionnels (Hutchins 1995). Cette interrogation implique une modification des unités d'analyse : on ne postule plus un centre de contrôle cognitif (linguistiquement parlant, il s'agirait des compétences langagières du locuteur), mais une distribution des agentivités cognitives dans les ressources de l'environnement. Dans cette perspective, comme le précise Lenay, "[...] les dispositifs techniques ne réalisent pas seulement des modifications artificielles d'une cognition qui serait "naturelle", mais ont un rôle constitutif des activités cognitives pour la perception, le raisonnement, la mémoire, l'imagination ou les interactions " (2001: 47). Les outils technologiques, considérés comme des agents psychiques dans le paradigme de la cognition distribuée, et dotés d'affordances selon la théorie de Gibson et Norman, participent donc à l'élaboration des discours.

$7 \quad$ Faute de place je me contente de mentionner les travaux en philosophie sur l'externalité de l'esprit (l'article fondateur de Clark et Chalmers, «The extended Mind» en 1998, les réflexions de Dennett également) : ils défendent l'idée que la conscience s'étend hors de notre esprit, et que nous pensons donc avec les autres esprits, mais aussi avec les éléments non humains de nos environnements. 
Ces approches ont en commun l'option postdualiste qui permet de dépasser l'analyse logocentrée des phénomènes discursifs et de considérer qu'il y a un continuum et non une rupture entre le linguistique et l'extralinguistique. Les observables de l'analyse ne sont plus seulement des éléments purement langagiers, mais des matières composites, métissées de social, de culturel, d'historique, etc., mais aussi de technologique. Ces observables sont le produit de ce que j'appelle une technologie discursive.

\subsection{Technologie discursive}

9 La technologie discursive est l'ensemble des processus de mise en discours de la langue dans un environnement technologique. C'est un dispositif au sein duquel la production discursive est intrinsèquement liée à des outils technologiques en ligne ou hors ligne (ordinateurs, téléphones, tablettes, logiciels, applications, sites, blogs, réseaux, plateformes). La notion est proposée à la suite de celle de technologie cognitive pour rendre compte, dans la perspective d'une cognition externe, de la contribution d'instruments matériels à l'élaboration des prédiscours (cadres préalables à la construction des discours). Elle se construit aussi par rapport à celle de technologie intellectuelle mise en place dans les années 1970 dans les études sur la transmission d'informations. Le terme de technologie intellectuelle est diffusé plus largement à partir des travaux de Goody sur l'écriture et connaitra une fortune importante en sciences de l'information et de la communication, surtout chez les chercheurs qui s'occupent d'Internet et des dispositifs numériques (chez Robert, Lévy, Ertzscheid, Gallezot notamment).

\subsection{Délinéarisation}

10 La notion de technologie discursive rend compte du fait qu'en ligne, les formes de discours sont co-constitutives des matières technologiques et des dispositifs communicationnels. Le genre de la "demande d'amitié» par exemple, issu de l'écosystème du réseau social Facebook ${ }^{2}$, est par définition composite, c'est-à-dire en même temps technologique et discursif. Cette demande passe en effet par un bouton (en français : "ajouter »), sur lequel il faut cliquer, en accompagnant (ou pas) ce clic d'un message scriptural. La demande d'amitié est donc une forme discursive constitutivement technodiscursive, dont il ne me semble guère possible de faire une analyse au sein d'une approche logocentrée : elle est délinéarisée, au sens où sa linéarité langagière, celle de la combinatoire de la phrase, est rompue et métissée de technique. Ce type de dispositif a pour fonction principale celle du Web 2.0 en général : la mise en relation.

11 On peut donc définir la délinéarisation comme une élaboration du fil du discours dans laquelle les matières technologiques et langagières sont co-constitutives, et modifient la combinatoire phrastique en créant un discours composite à dimension relationnelle.

12 La délinéarisation concerne également de nombreuses formes discursives en ligne, à commencer par les liens hypertextes: la profondeur qu'apporte le lien comme forme cliquable ouvrant sur un autre texte délinéarise l'énoncé. Autre exemple, le tweet, qui est l'énoncé délinéarisé par excellence, puisqu'il contient des formes technolangagières (éléments langagiers cliquables permettant la relation avec un autre plan discursif) : des technomots comme le hashtag (précédé de \#) et le pseudo (précédé de @), ainsi que des liens (qui peuvent être doublement technolangagiers dans la mesure où ils peuvent être 
raccourcis par des applications dédiées). Les tweets peuvent également contenir des émoticones, technoformes anciennes et désormais bien connues, mais également d'autres types de symboles, comme des notes de musique, des étoiles, des soleils et des formes graphiques issues de l'art ASCII (pour une synthèse sur les dispositifs linguistiques de Twitter, voir Paveau 2013b).

Fig. 1. Délinéarisation. L'exemple des tweets (hashtags, pseudos, liens entiers, liens raccourcis)

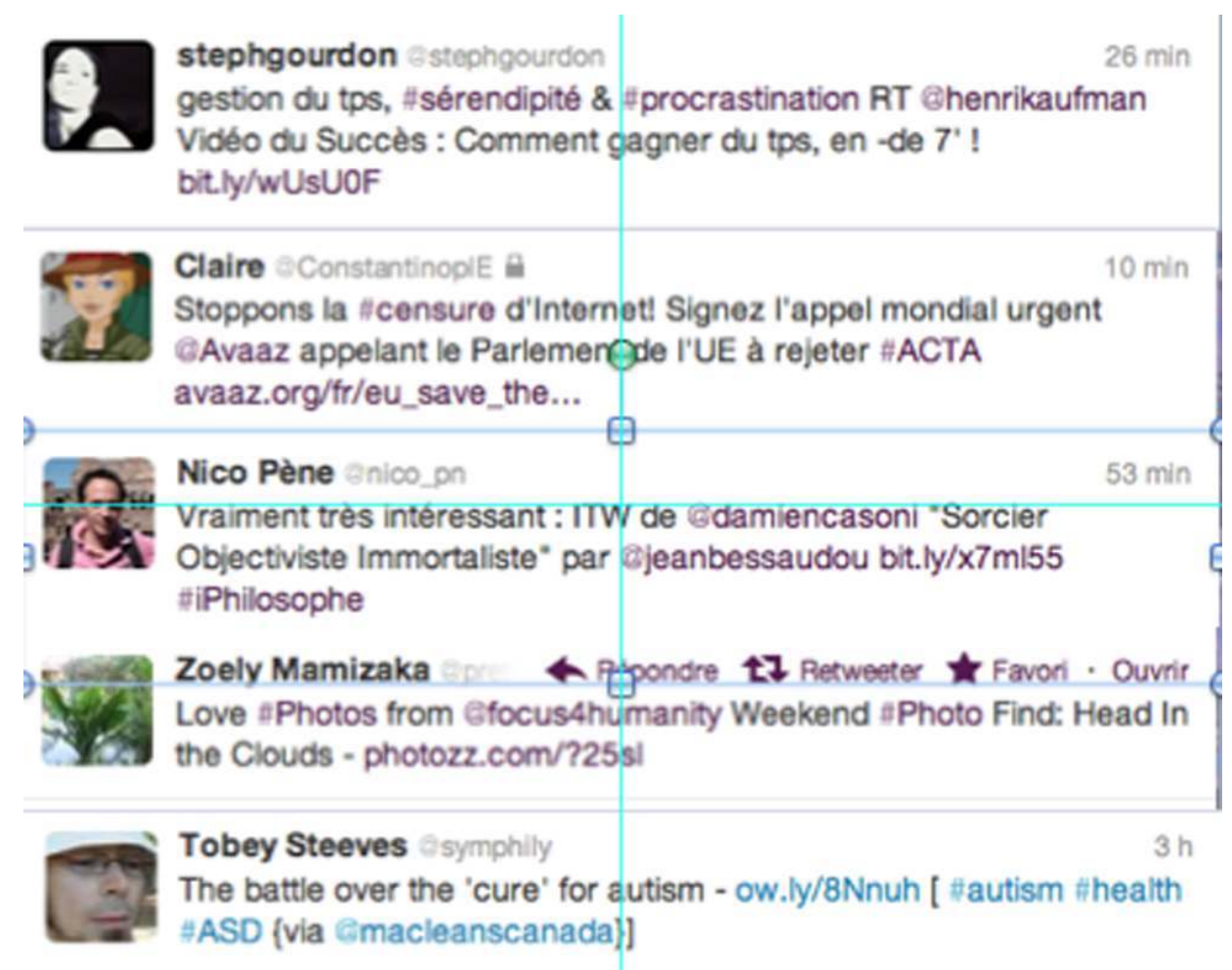

\section{Les trois ordres linguistiques du numérique}

Le terme numérique est devenu générique et recouvre des situations technodiscursives différentes : entre un texte scanné non modifiable et simplement mis en ligne, et un billet de blog avec de nombreux hyperliens, il existe des différences d'ordre linguistique qui concernent le mode de production de l'écrit, le mode de lecture du texte et son inscription dans l'écosystème du Web. Je propose une typologie à trois entrées qui repose sur des critères linguistiques : numérisé, numérique, numériqué. Cette typologie correspond globalement à la tripartition que Dacos et Mounier proposent dans leur ouvrage L'Édition électronique (2010). Ils soulignent en effet qu'il s'agit d'un domaine rassemblant trois pratiques différentes: la numérisation comme "portage du texte imprimé », l'édition numérique qui exploite "nativement les possibilités qu'offre le texte numérique » et l' édition en réseau qui développe « de nouveaux modes de production des textes » (Dacos et Mounier 2010 : 6-7).

14 Cette typologie, qui concerne la chaîne éditoriale, prend surtout en compte le type de support et son mode de création et de diffusion. Celle qui suit essaie de rendre compte des traits langagiers et discursifs des textes produits dans les contextes numériques. 


\subsection{Numérisé}

15 Un document numérisé est le produit d'un portage dans un environnement numérique. À partir d'une version imprimée, il est entré dans un logiciel (par exemple Hyperbase, fig. 2), ou scanné (par exemple sur la base Persée, fig. 3 et 4). Il est mis en ligne ou non.

Fig. 2. Hyperbase, extrait de Mayaffre 2005

Figure 3: Lecture parallèle du texte brut (partie gauche) et du texte lemmatisé (partie droite) dans Hyperbase (ici allocution de de Gaulle, le 13 juin 1958).

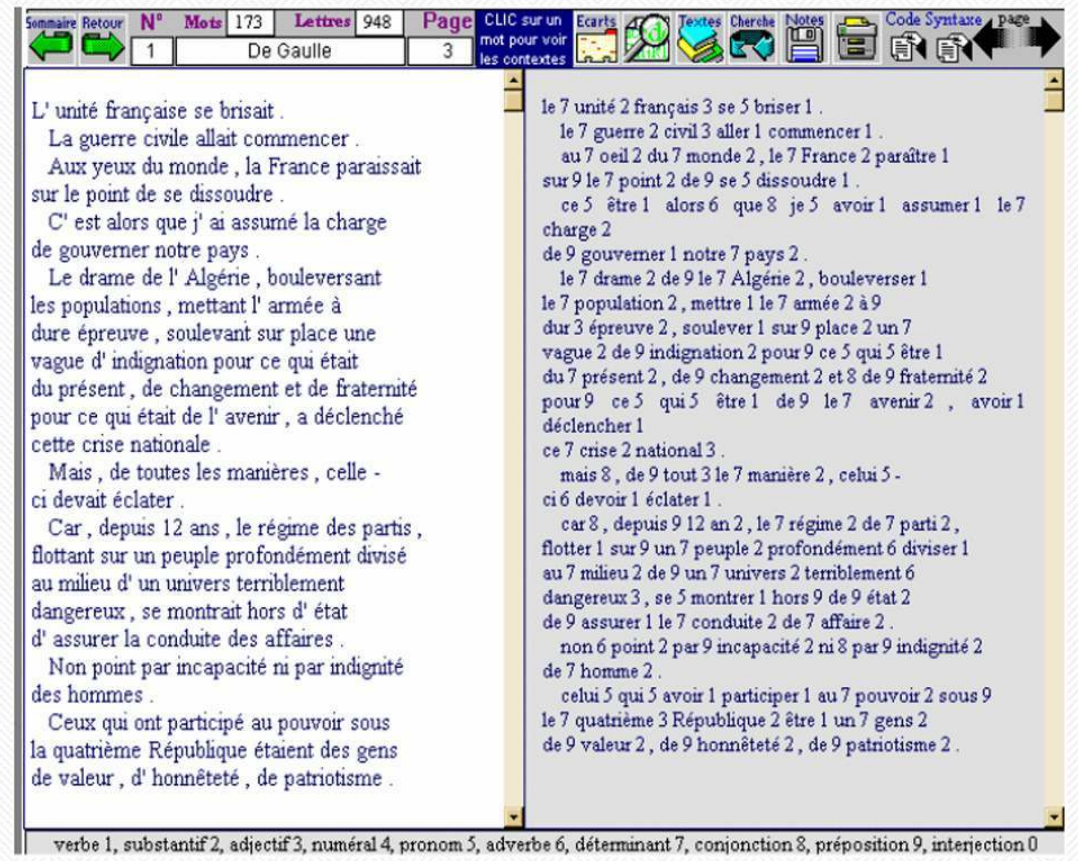

Il est plus ou moins navigable et modifiable : Hyperbase et Lexico 3 permettent évidemment de naviguer dans les textes et de les modifier puisqu'il s'agit d'outils destinés à l'analyse des textes, mais les textes de Persée par exemple, téléchargeables en format PDF, permettent seulement une navigation restreinte (recherche de mots-clés), sans modification possible du texte, si ce n'est par annotations externes via les possibilités logicielles d'Acrobat Reader ou d'autres lecteurs de fichiers PDF (fig. 3 et 4). Les configurations de navigabilité et modifiabilité sont diverses et dépendent des affordances des environnements (Paveau 2012b) : Google books, qui ne permet pas de manipulation des textes numérisés, permet cependant une navigabilité hypertextuelle dans les tables des matières et une recherche par mots-clés. On peut distinguer une modifiabilité interne (modifier le texte) et une modifiabilité externe, c'est-à-dire de type éditorial (modifier la police, la couleur et la taille de la police, le fond, etc., ce que permettent les liseuses par exemple). Mais il faut préciser que la modifiabilité dépend du format plus que du support: une liseuse qui permet de lire du PDF, ne permettra pas plus de le modifier qu'un autre support. Elle permettra en revanche de l'annoter, ce qui est une autre forme de modifiabilité3. 
Fig. 3. L'introduction du numéro 1 des Actes de la recherche en sciences sociales en ligne

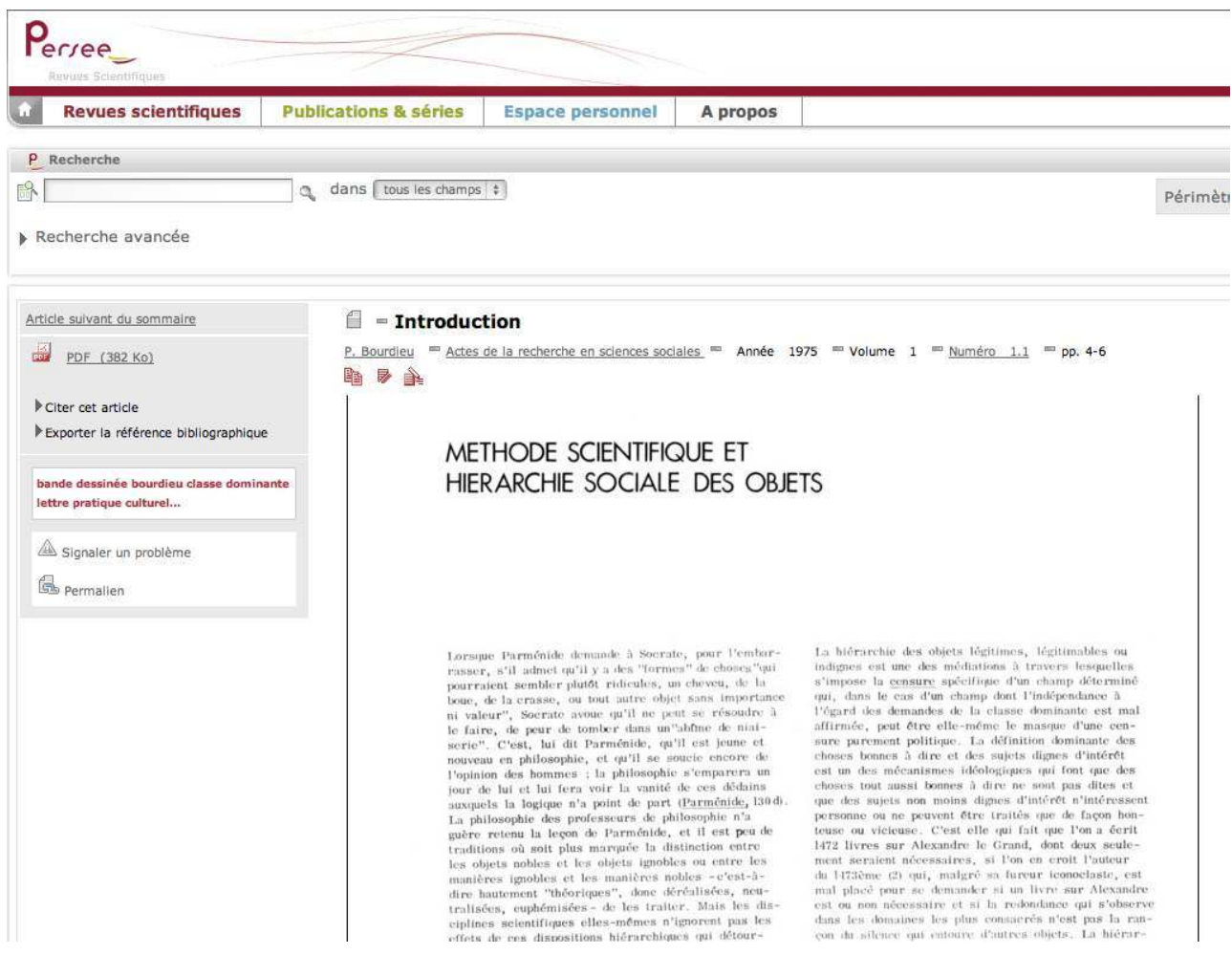

Fig. 4. L'introduction du numéro 1 des Actes de la recherche en sciences sociales en PDF téléchargé

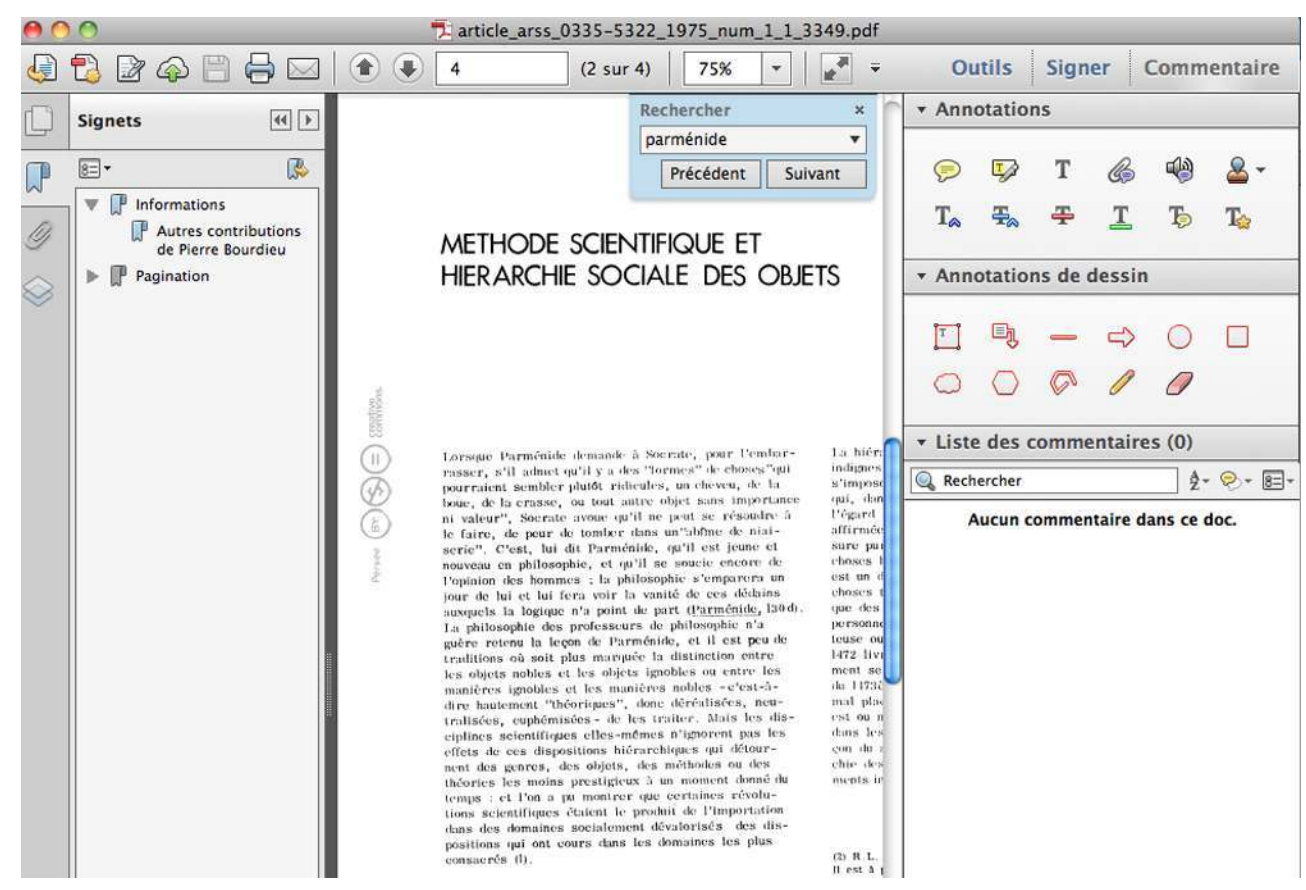

Le texte numérisé ne comporte pas de traits technolangagiers, c'est-à-dire d'éléments (mot, segment, phrase) cliquables qui mèneraient à d'autres documents en ligne par le biais technodiscursif. Il ne comporte pas non plus de technosignes ${ }^{4}$ (par exemple des boutons de partage sur les réseaux sociaux ou les sites de bookmarking) qui permettraient la circulation ou l'archivage sur un autre espace, en ligne. Il ne permet pas 
le commentaire ou la conversation, ce qui témoignerait d'une énonciation augmentée, et ne relève pas non plus des technogenres du Web qui lui sont natifs. Il n'est de ce fait pas intégré dans l'écosystème du Web, même s'il est mis en ligne. C'est un texte qui effectue un simple passage du support de l'impression sur papier au support électronique. On pourrait dire que l'imprimé est dans la « nature » du texte, et qu'il s'y maintient (même si cet imprimé a été, dans sa première vie, un fichier électronique).

\subsection{Numérique}

Un document numérique est produit en contexte électronique hors ligne, sur un ordinateur, un téléphone, une tablette, et il possède toutes les caractéristiques de l'écriture au clavier et les fonctions apportées par les affordances du logiciel d'écriture. Il est facultativement mis en ligne (livres numériques, revues mises en ligne) et peut donc s'intégrer dans l'écosystème du Web ou rester hors ligne (fichiers de travail); mais il n'est pas nativement destiné à la mise en réseau. Il peut comporter des technomots et des hyperliens que les logiciels d'écriture permettent désormais de créer mais non des technosignes.

Sauf artifice non natif du système (colorer à la main les interventions des locuteurs), les documents numériques ne permettent pas une énonciation augmentée ou écriture à plusieurs prévue sur les pads par exemple ${ }^{5}$. En revanche un texte produit hors ligne et mis en ligne peut être intégré dans un dispositif d'énonciation augmentée, comme dans le cas de l'open peer review: dans l'exemple de la figure 5 , les textes présentés sont tous sous Word ou PDF, et c'est leur rassemblement sur la page qui constitue une augmentation de l'article initial. Les genres de discours restent ceux des contextes hors ligne puisque les technogenres se définissent par leur inscription dans les environnements du Web. Les textes sont navigables et modifiables en interne et en externe sauf verrouillage; ils sont annotables. Enfin, ils sont imprimables, comme le montrent les icônes d'impression sur les sites des revues en ligne par exemple : il peut y avoir en effet passage à l'imprimé sans que l'imprimé ne soit dans la "nature" du texte numérique; on pourrait dire que l'imprimé peut facultativement faire partie du devenir du texte numérique sans qu'il ne constitue son destin. 
Fig. 5. Le système d'open peer review de la revue BMC Medicine

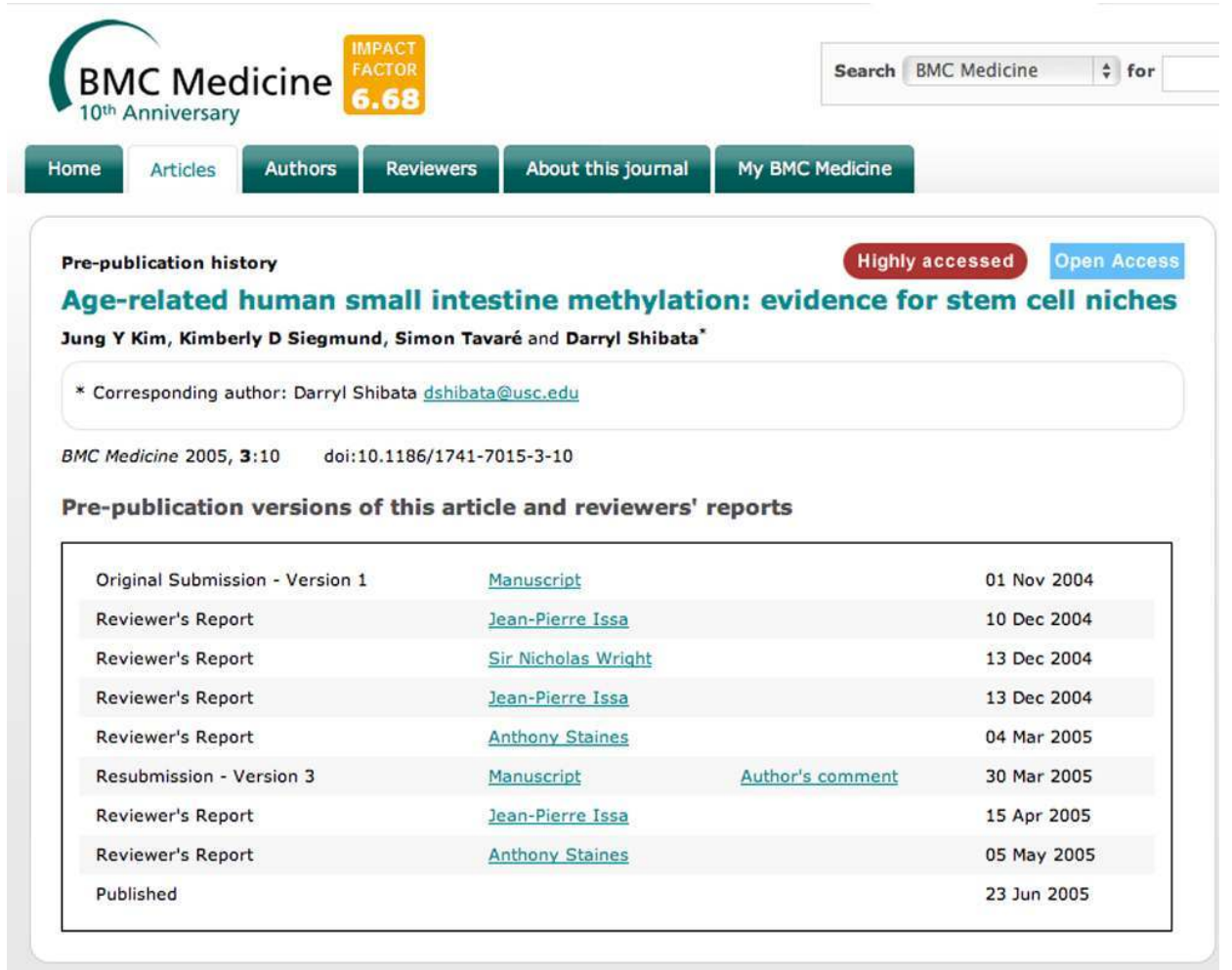

\subsection{Numériqué}

Un document numériqué est produit nativement en ligne, sur un site, un blog ou un réseau social, tout lieu numérique accueillant de la production de discours. Il présente des traits de délinéarisation du fil du discours, d'augmentation énonciative, de technogénéricité et de plurisémioticité6.

- Délinéarisation. Le fil du discours est nativement délinéarisé par des technomots, technosignes et hyperliens (fig. 1). Cette délinéarisation n'est cependant pas obligatoire puisque dans certains contextes, liés à des genres, les scripteurs en ligne écrivent de manière linéaire, par exemple sur certains blogs littéraires ou sur Twitter dans le cadre de la twittérature (Paveau 2013a) : le texte ressortit alors à la catégorie « numérique ».

- Augmentation. Dans les contextes numériques, la prérogative énonciative n'est plus réservée à une figure unique, l'énonciateur, comme le veulent les approches mainstream actuellement en sciences du langage, où l'on parle d'énonciateur comme source du discours, même si l'on admet qu'il puisse s'agir d'un individu unique comme d'un collectif voire d'une source non identifiée ou même vide comme pour la rumeur ou le proverbe. Le schéma de l'énonciation issu des travaux de Benveniste en particulier, comprenant l'énonciateur, l'énonciataire, le temps et le lieu de l'énonciation, est toujours en vigueur même s'il est davantage question désormais de co-énonciation (Culioli 1990) rendant mieux compte du circuit complexe de l'énonciation et surtout de la complexité de la notion de source énonciative. Mais la question reste toujours : «Qui parle? » En contexte numériqué, cette question a perdu de sa pertinence et la notion d'énonciateur doit être révisée via celle d'augmentation car les énonciateurs ont des voix composites elles aussi. 
Sur un blog, un billet, signé d'un "auteur ", sera augmenté par les discussions : au bout du compte, qui sera l'énonciateur du billet? Sur un pad, plusieurs locuteurs, identifiés par des marques graphiques (couleurs), rédigent ensemble, ce qui change un peu la donne énonciative (fig. 10) : d'une part la visibilité de cette énonciation multiple est propre à ce type d'outils et ne se retrouve pas dans les textes rédigés de manière plus traditionnelle, et d'autre part peut-on encore parler de source énonciative identifiable? On a là une augmentation étroitement dépendante des affordances de l'environnement: on est bien dans du techno-énonciatif, qui ne permet plus une approche égocéphalocentrée (Brassac 2010).

- Technogénéricité. Les environnements technodiscursifs ont permis l'apparition de genres de discours qui leur sont dépendants. Tous les genres de discours du Web ne sont pas spécifiques (voir sur cette question Maingueneau 2013), mais certains d'entre eux ne peuvent exister qu'en ligne à cause des traits technologiques qui les définissent. C'est par exemple le cas de la twittérature sur le réseau de microblogging Twitter (Paveau 2013a), qui suppose l'environnement technologique du réseau ou de la demande d'amitié Facebook mentionnée plus haut. Comme genre de discours, la demande d'amitié n'existe pas chez les adultes dans les rituels sociaux hors ligne, mais elle est pratiquée par les enfants comme le montrent le titre de cet album d'Éric Battut, Veux-tu être mon ami ? (Didier-Jeunesse, 2009) ou les échanges ordinaires que l'on peut capter par exemple sur les forums de jouets comme OhMyDollz. La demande d'amitié entre adultes peut donc être considérée comme une sorte d'invention du Web social, comme le précise Casilli :

Les structures d'une société en ligne, dès lors, ne se limitent pas à un décalque fidèle des logiques et des processus sociaux que nous pouvons observer l'ordinateur étant éteint. L'entraide, la coopération et la proximité des individus et des groupes prennent des formes originales dans le contexte des médias numériques. (2010:59)

La demande d'amitié est véritablement un technogenre dans la mesure où elle passe nativement et obligatoirement par le bouton "ajouter " (fig. 11). Les formes peuvent varier, de la requête technodiscursive brute par simple clic sur le bouton (ce qui se produit si l'abonné Facebook empêche l'envoi de messages par exemple) à la demande assortie d'un message selon le style des réseaux sociaux (pour aller vite : informel) ou selon le style épistolaire du courrier électronique ou de la lettre hors ligne (pour des exemples, voir Paveau 2012a).

- Plurisémioticité. Délinéarisation, augmentation et technogénéricité peuvent être assorties d'une plurisémioticité : en ligne, les productions discursives peuvent contenir des images de toutes sortes, des vidéos, des enregistrements sonores, etc.

Pour terminer, revenons au rapport papier-numérique. Le texte numériqué est-il imprimable ? Y a-t-il un «retour » au papier possible? A priori, les traits précédemment analysés impliqueraient que l'imprimé n'est pas dans la nature du texte et ne fait pas partie de son devenir : on n'imprime ni les technomots, ni les vidéos, ni les énonciations augmentées. Il existe cependant des expériences de passage de textes nativement numériqués à des livres imprimés. C'est le phénomène que saisit Vignola dans Du blogue au livre. Réflexions sur la nature générique du blogue. Il se situe dans une perspective différentialiste car pour lui, le mode de lecture du blog est très différent de celui du livre : " même les gens qui ne lisent jamais savent lire un livre (où se trouvent le début et la fin, comment tourner les pages, etc.). Le lecteur de blogue doit découvrir (ou inventer) une nouvelle logique de lecture » (2009: 13). Du coup, la publication papier d'un blog présente des traits particuliers qui visent à combler les écarts entre les deux types de publication. 
Vignola analyse en particulier les trois blogs publiés, en 2007 aux Éditions du Septentrion, dans la collection «Hamac-carnets »: Les chroniques d'une mère indigne de Caroline Allard, Un taxi la nuit de Pierre-Léon Lalonde et Lucie le chien de Sophie Bienvenu. Vignola souligne qu'il existe

deux manières de publier un blogue sur support papier: la première, plus répandue, consiste à rapatrier du texte de blogue dans un genre déjà établi (le roman, le journal, l'essai, etc.) ; la seconde, plus récente, fait le pari de publier le blogue comme un genre nouveau, sans tenter de le lier à quelque chose de plus établi. (2009 : 25-26)

Les points de réflexion de Vignola concernent essentiellement les commentaires, les menus (et autres métadonnées présentes sur les blogs) et l'ordre antéchronologique, respecté dans certaines publications.

Autre exemple de ce passage du numérique au papier: Zythom, le blog d'un informaticien expert judiciaire, blog célèbre qui compte désormais plus de 700 billets, connaît depuis quelque temps une expérience analogue. Le blogueur vient de terminer le quatrième tome de son blog, les trois premiers sont disponibles (fig. 6 et 7).

Fig. 6. Zythom le blog

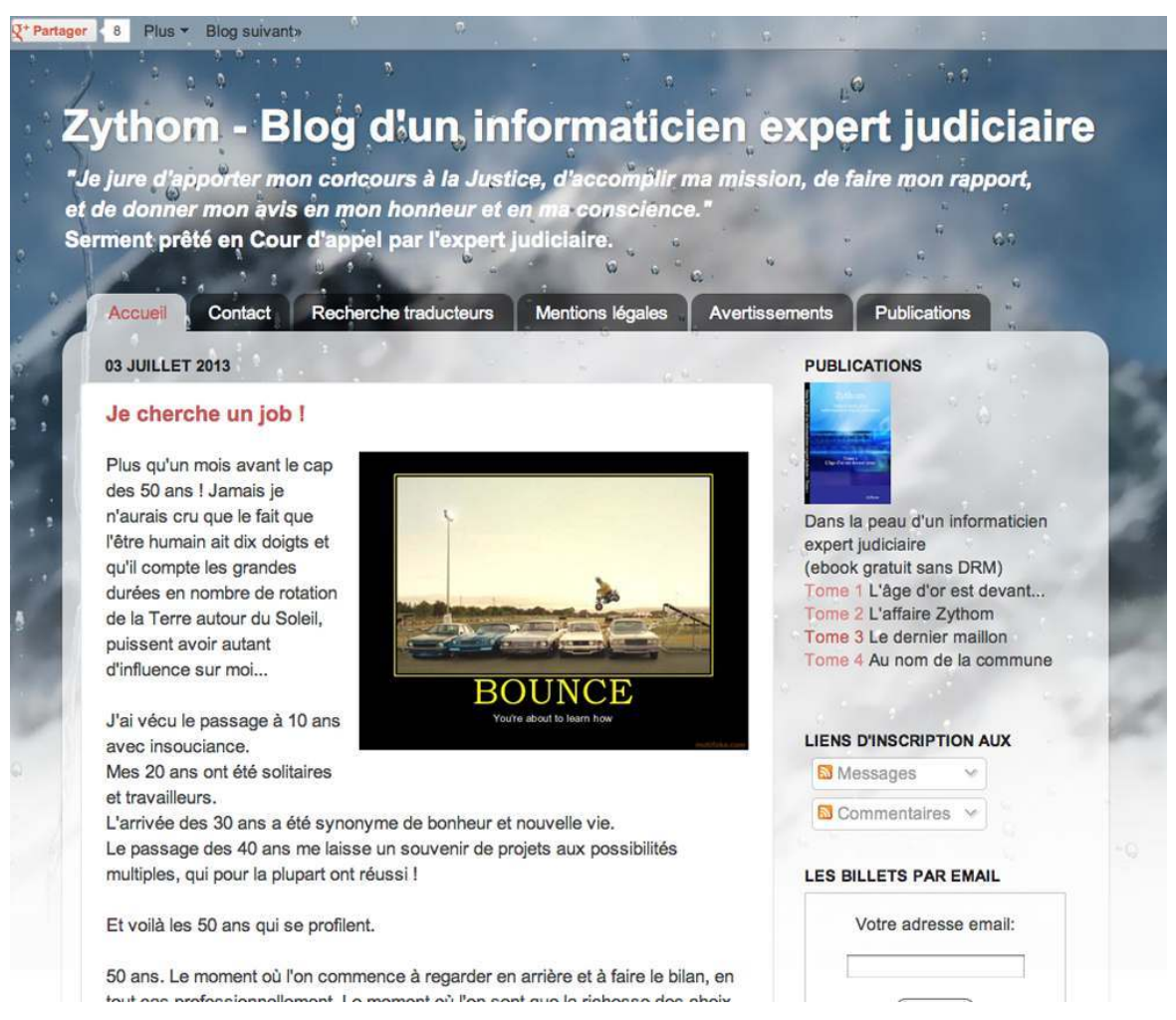




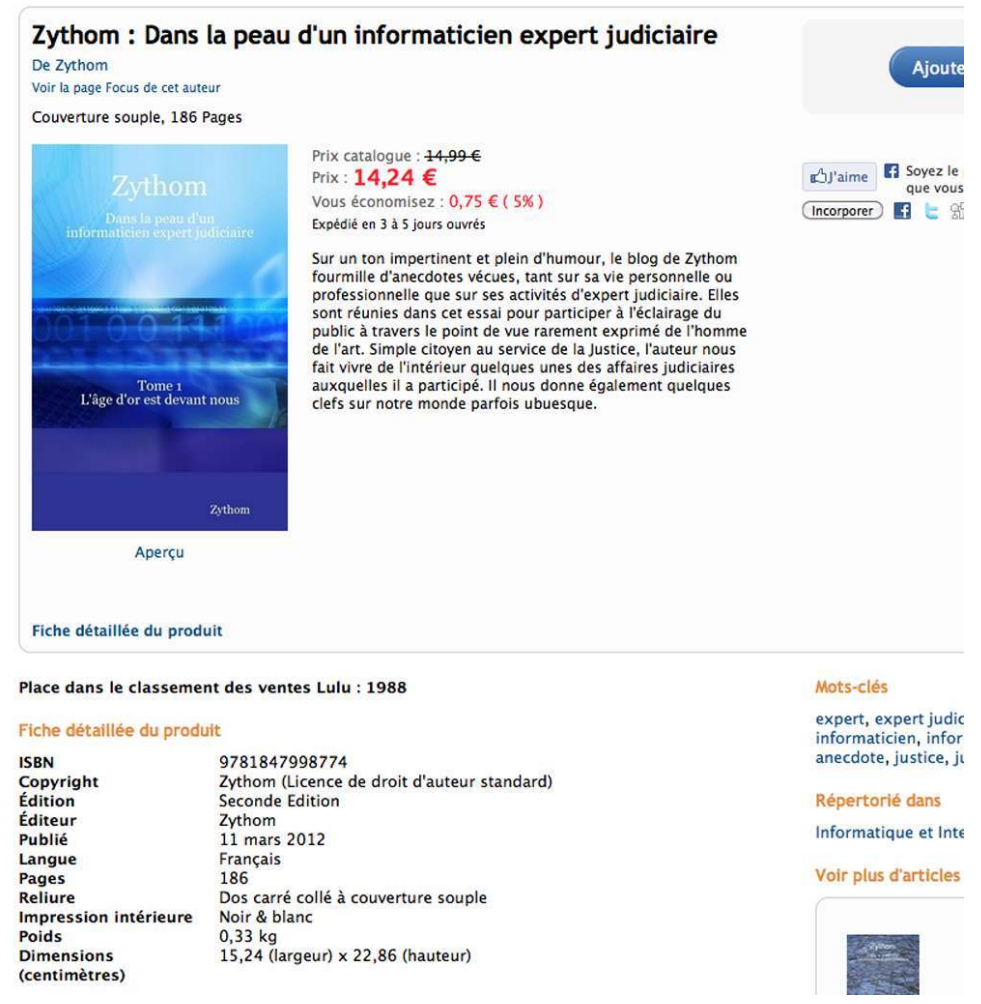

Tous sont publiés en auto-édition et, outre le format papier traditionnel, ils existent en six formats numériques gratuits (PDF, EPUB, FB2, LIT, LRF, MOBI). Zythom décrit peu les transformations linguistiques et sémiotiques des billets passés aux chapitres mais note cependant :

Le passage d'un billet de blog à une version papier nécessite la suppression des liens. J'ai donc inséré beaucoup de "notes de bas de page" pour expliquer ou remplacer les liens d'origine. Dans la version électronique, j'ai laissé les liens ET les notes de bas de page. (28 avril 2012 ; graphie conservée)

Il est intéressant de voir que dans l'esprit de ce blogueur, lien et note de pas de page sont à peu près équivalents, en tout cas dans le contexte de ce changement d'environnement sémiotique. Sur les versions électroniques, l'auteur a numériqué tous les titres de chapitres qui correspondent aux billets, et conservé des liens, assez rares, dans le corps du texte: les fichiers proposent donc une version encore délinéarisée, qui correspond assez bien au format que j'appelle numérique, médian entre numérisé et numériqué.

\section{Aspects énonciatifs de l'identité numérique}

Les linguistes, qui pensent que tout passe par le langage, et qui ont souvent raison, auraient envie de définir l'identité numérique comme la forme du sujet de l'énonciation en ligne, c'est-à-dire son pseudo, son avatar, sa signature. Cette conception est réductrice et l'identité numérique recouvre un ensemble de phénomènes bien plus large. La notion est amplement traitée dans les disciplines qui s'intéressent à la présentation de soi sur le Web: sciences de l'information et de la communication, sociologie, philosophie, intelligence artificielle, droit, humanités numériques. L'identité numérique est l'ensemble des données personnelles que nous déposons ou laissons à notre insu sur le Web, c'est-à- 
dire ce que nous faisons, disons, partageons, ressentons, aimons, détestons, recherchons, etc. C'est l'ensemble de nos traces numériques, des marques de notre présence en ligne. Si elle est toujours sémiotisée, elle n'est donc pas forcément verbale: un like, une inscription à un site, une commande de livre ou une réservation de billet, un paiement par carte, la publication de photos constituent autant d'éléments de notre présence en ligne que les discours langagièrement articulés que nous tenons. Je détaille ici trois aspects énonciatifs des identités numériques: le pseudonymat, la technologie du soi et l'augmentation.

\subsection{Identités pseudonymes}

30 Sur le plan langagier, il est un élément fondamental dans l'ensemble de nos traces : notre nom. Et sur le Web, nous sommes identifiables par des noms qui peuvent être de faux noms, des pseudonymes. Nous pouvons tout à fait vivre en ligne sous notre nom d'état civil, mais nous pouvons aussi choisir un autre nom, pratique qui appartient pleinement aux normes de présentation de soi en ligne. Sur le Web, «nobody knows you are a dog », comme le dit un dessin devenu proverbial.

31 Le pseudonymat est le choix par un sujet d'un «falsum nomen ", un « nom fait à plaisir » selon l'expression de Laugaa (1986). Martin a consacré un ouvrage au pseudonymat en ligne en sciences du langage, Se nommer pour exister (2012), qui prolonge et développe celui de 2006, Le Pseudonyme sur Internet, une nomination située au carrefour de l'anonymat et de la sphère privée (Martin 2006). Un article de Cislaru paru en 2009, « Le pseudonyme, nom ou discours ", traite la question sous l'angle sémantique et discursif. Martin s'appuie sur le travail littéraire de Laugaa dans La Pensée du pseudonyme (1986), qui porte surtout sur la question de l'auteur. En linguistique, on peine à trouver des travaux, sans doute parce que l'objet légitime de la linguistique serait plutôt l'anthroponyme.

Dans Se nommer pour exister, Martin propose plusieurs enquêtes: la construction des pseudonymes dans les adresses courriel, sur les forums et les salons de discussion, et l'analyse de pseudonymes recueillis sur des forums et blogs constituant huit corpus. Ils sont mentionnés dans ce tableau de synthèse : 
Fig. 8. Tableau récapitulatif des huit corpus de travail de Martin (2012)

\begin{tabular}{|c|c|c|c|c|}
\hline \multicolumn{5}{|c|}{$\begin{array}{l}\text { Tableau } 66 \text { - Étude chiffrée des huit corpus présentés dans le } \\
\text { présent chapitre }\end{array}$} \\
\hline \multicolumn{5}{|c|}{ Corpus 1: De la maladie et des pseudonymes } \\
\hline En chiffre & \multicolumn{2}{|c|}{$\begin{array}{l}\text { Sphère privée du } \\
\text { sujet en chiffre et } \\
\text { en } \%\end{array}$} & \multicolumn{2}{|c|}{$\begin{array}{l}\text { Cryptonymes en } \\
\text { chiffre et en } \%\end{array}$} \\
\hline 150 & 87 & $57 \%$ & 63 & $43 \%$ \\
\hline \multicolumn{5}{|c|}{ Corpus 2 : De l'environnement et des pseudonymes } \\
\hline 200 & 117 & $59 \%$ & 83 & $41 \%$ \\
\hline \multicolumn{5}{|c|}{ Corpus 3: De l'identité nationale et des pseudonymes } \\
\hline 200 & 137 & $69 \%$ & 63 & $31 \%$ \\
\hline \multicolumn{5}{|c|}{ Corpus 4: Des jeux vidéo et des pseudonymes: le cas Nintendo } \\
\hline 150 & 88 & $54 \%$ & 62 & $46 \%$ \\
\hline \multicolumn{5}{|c|}{ Corpus 5 : Blocs-notes du journal en ligne : Le Figaro } \\
\hline 60 & 40 & $67 \%$ & 20 & $33 \%$ \\
\hline \multicolumn{5}{|c|}{ Corpus $6:$ Blocs-notes du journal en ligne : Le Monde } \\
\hline 100 & 72 & $72 \%$ & 28 & $28 \%$ \\
\hline \multicolumn{5}{|c|}{$\begin{array}{c}\text { Corpus } 7: \text { Pseudonymes dans les commentaires de la presse en } \\
\text { ligne : le magazine Le Point }\end{array}$} \\
\hline 145 & 81 & $56 \%$ & 64 & $44 \%$ \\
\hline \multicolumn{5}{|c|}{$\begin{array}{c}\text { Corpus } 8: \text { Pseudonymes dans les commentaires de la presse en } \\
\text { ligne : MSN }\end{array}$} \\
\hline 150 & 112 & $75 \%$ & 38 & $25 \%$ \\
\hline
\end{tabular}
issu de l'espace privé du sujet est majoritaire. Les corpus de Martin montrent en effet que le pseudonyme, s'il masque une identité d'état civil en œuvre dans la vie sociale hors ligne, est au cœur d'une riche élaboration sémantique de soi en ligne. La faible proportion des pseudonymes cryptonymiques confirme la fonction communicationnelle du falsum nomen. Le pseudonyme apparaît alors comme le lieu d'un «véritable» fragment d'identité.

37 - Un nom unique sans homographe possible. C'est sans doute la grande différence avec le nom propre "IRL" (in real life) : en ligne, sur un écosystème donné (un site, un réseau, correspondant à une base de données) aucune homonymie n'est possible. Impossible de s'appeler Dupont ou Durand en ligne, sauf à y ajouter une numérotation :

Si la création pseudonymique est pléthorique sur le Web, il n'en reste pas moins que l'homonymie est impossible dans une même base de données. Fonctionnant avec un code binaire de type oui/non, le langage informatique ne permet pas 
l'homonymie, puisqu'elle est alors considérée comme une seule et même information. (Martin $2012: 112$ )

38 Au contraire des idées reçues souvent négatives sur l'anonymat en ligne, qui insistent sur la confusion, la désidentité et la dissimulation, il ne peut y avoir que des noms uniques, bien plus faciles à retrouver que les noms fréquents.

\subsection{Une technologie technolangagière de soi}

J'aimerais compléter les résultats de Martin en faisant appel au concept de «technologie du soi » chez Foucault. Foucault commence à aborder cette notion au début des années 1980 (Foucault 1982, 1983, 1984), en dehors d'une référence aux univers discursifs numériques bien sûr, le mot technologie étant pris au sens de procédure ou de méthode. Pour rendre compte de la dimension numérique, je parlerai donc plutôt de «technologie technolangagière du soi ». D'une manière plus générale, on parle de technologie numérique du soi, comme le font Dervin et Abbas (2009). Foucault définit les technologies du soi ou techniques de soi comme les «procédures [...] qui sont proposées ou prescrites aux individus pour fixer leur identité, la maintenir ou la transformer en un certain nombre de fins et cela grâce à des rapports de maîtrise sur soi et de connaissance de soi » ([1982] $2001: 1032)$.

40 Il me semble que le pseudonyme en ligne constitue une technique pour cette technologie du soi : c'est une unité langagière à laquelle se sont ajoutés des traits spécifiques au Web, mais également propres à ce contexte poststructuraliste où les identités ont perdu leur fixation et leur solidité pour devenir multiples et liquides, selon l'expression de Bauman. L'usage du langage en ligne n'est pas inédit, mais prend des traits inédits, qui s'ajoutent aux formes hors ligne sans les effacer (cas des technomots par exemple). Ces traits sont langagiers mais également sociodiscursifs, engageant la technologie langagière du soi et la modernité réflexive de l'individu dans de nouvelles formes identitaires marquées par la fluidité. J'en propose quatre.

\subsubsection{Un lexème inédit : métissages morphologiques}

41 Le pseudonyme est un lexème inédit sur le plan morphologique : il s'agit d'un nom propre qui peut emprunter, sans procédure historiquement longue de lexicalisation (comme c'est le cas des noms communs devenant noms propres IRL du type Boulanger ou Lecouvreur) des traits de nom commun, et même d'expression ou de phrase, mais aussi des signes typographiques non verbaux (tirets, ponctuation), des chiffres (formes du langage SMS) et des formes de métissage interlinguistique. Ce métissage morphologique n'appartient pas aux univers identitaires hors ligne ; en voici quelques exemples, tirés des corpus de Martin (sauf la dernière série) :

- éléments langagiers et chiffres : kooky64, sol94, maba12323 ;

- éléments langagiers et signes typographiques : malmo[FR], [M-W_2];

- phrases : help-me-please99, mon mal de vivre (et sous une autre forme : mal 2 vivre), The-heart-ofa-girl-broken, tomatesdelatreille, l'étoile de la délivrance ;

- métissage interlangue : lekingdu16, The_Virtuose, le howaway (hors tableaux ci-dessus).

À l'extrême contrainte du nom unique semble s'opposer l'absolue liberté morphologique des sujets en ce qui concerne leur auto-nomination. Cislaru (2009) signale à juste titre qu'il existe de nombreuses pages de conseils voire de consignes pour l'élaboration du 
pseudonyme en ligne ; on trouve également des générateurs de pseudos en ligne ainsi que des parodies de guides donnant en négatif les « bonnes » règles pour les élaborer. Il s'agit bien d'une pratique discursive installée dans l'écosystème du Web.

\subsubsection{Fluidité d'un sémantisme environnemental}

Le pseudonyme est sémantiquement évocateur. Il a, en termes sémantiques, une signifiance ou un feuilleté sémantique (Lecolle et al. dir., 2009). Considéré dans les théories mainstream comme un désignateur rigide il est, dans les théories alternatives, ce que j'appelle un « désignateur souple ». L'élaboration sémantique est contextuelle, et tous les agents de l'environnement y contribuent. Je donne un bref exemple personnel : quand j'ai ouvert mon compte Twitter, mes initiales map étaient prises, mapav aussi et j'ai donc choisi mapav8 en ajoutant un chiffre qui est l'un des marqueurs de ma biographie. Je suis donc @mapav8, ce qui est un pseudo court qui dit des fragments de mon nom. Pour certains de mes correspondants, le 8 est celui de l'université Paris 8 et sur Twitter je figure sur une liste d'enseignants de Paris 8. Exemple intéressant qui montre bien à quel point l'élaboration du sens sur le Web est collective, partagée, fluide en un mot, et en aucun cas maitrisée par l'intentionalité de son producteur: le pseudonyme est environnemental, dépendant de l'ensemble des données numériques du porteur.

\subsubsection{Une catégorie sans autonomie : anonymat, pseudonymat, hétéronymat}

Si le pseudonyme est une catégorie en soi sur le plan de sa formation linguistique, elle ne peut être traitée de manière autonome: dans les univers discursifs numériques, il faut envisager un dispositif où pseudonymat, anonymat et hétéronymat ${ }^{9}{ }^{\prime}$ 'articulent les uns aux autres. On ne comprend pas grand-chose au pseudonymat sur Internet si l'on ne considère pas que le pseudonymat y est l'anonymat, ou, pour dire les choses autrement, que l'anonymat n'y existe pas. Aucun système ne permet en effet de ne pas signer une intervention : on laisse toujours au moins la trace de son adresse IP. Il existe d'ailleurs des rituels autour de cette question. Sur certains sites, si l'on ne donne pas de nom, falsum ou verum, une signature apparaît automatiquement: "Anonymous Coward", comme sur Slashdot, site d'actualités sur les technologies de l'information (fig. 9). 
Fig. 9. Exemple de commentaires automatiquement signés « Anonymous Coward » sur Slashdot

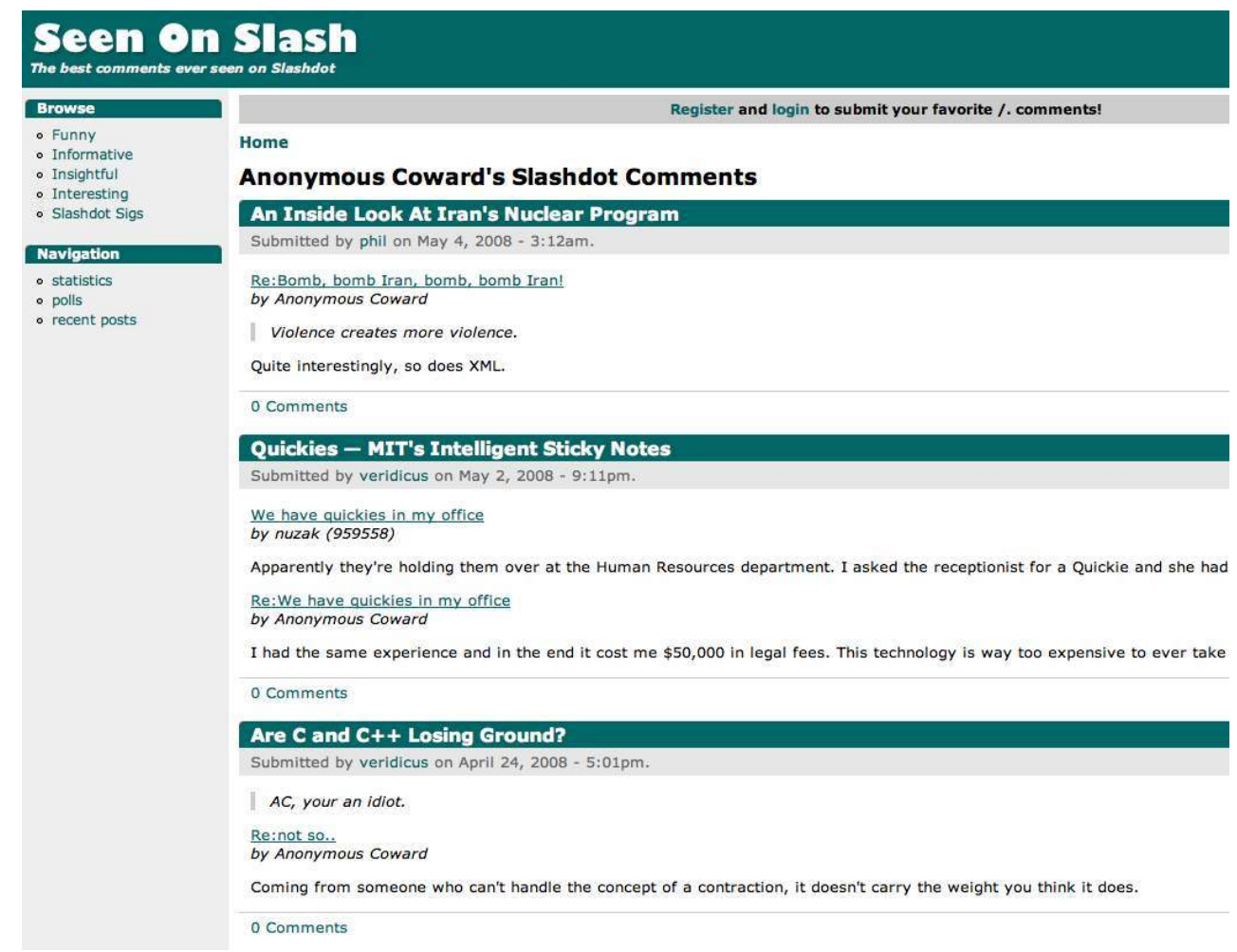

\subsubsection{Le pseudonyme dans le (dés)ordre du discours} en l'anonymat en ligne : en juin 2010 le sénateur français Jean-Louis Masson dépose une proposition de loi pour lever l'anonymat des blogueurs et en 2011, Google entend suspendre les comptes Google + sous pseudonymes. Ces affaires ont constitué un véritable événement discursif qui a reconfiguré le sens des mots anonymat et pseudonymat. Maître Eolas défend aussitôt les masques discursifs que sont les pseudonymes (2010), soulignant que nous sommes ce que nous disons sur le Web, et non des individus aux identités «solides", aurait dit Bauman. Sur le plan linguistique, cette remarque est intéressante car elle modifie la notion même de sujet énonciateur identifiable en déplaçant la portée de la prise de parole sur les discours plus que sur leurs énonciateurs. Sur ce point, les analyses des linguistes restent peu informées : on lit ainsi dans le Manuel d'analyse du Web que les blogs se caractérisent par «l'effacement de la source énonciative », en vertu d'une 
"opération de masque d'une source énonciative ", et les porteurs de pseudos y sont appelés «énonciateurs fictifs» (Temmar 2013: 94 et 96). La question d'une énonciation sans sujet y est même posée. Mais la question de la "source » énonciative, si tant est qu'elle soit clairement saisissable dans les identités «solides » du monde IRL, doit être repensée sur le Web, et non interprétée comme masquage et fictionnalité : un discours sous pseudonyme témoigne bien d'une présence en ligne, aussi réelle et habitée que le corps de chair et d'os qui est derrière le clavier, comme le montre boyd ${ }^{10}$ qui explique que pour des communautés minoritaires, opprimées ou même peu lettrées, le pseudo est une autorisation à la prise de parole en même temps qu'une protection (boyd 2010). En termes plus linguistiques, on pourrait dire que le pseudonyme est une autorisation au discours, une entrée dans la discursivité qui ne serait pas forcément possible sous identité officielle : c'est une condition de possibilité du discours.

\subsection{Augmentation énonciative : collectivité et multiplicité}

Les univers numériques nous amènent à écrire beaucoup, dans des lieux différents, sous des modalités énonciatives inédites, au sein de genres de discours et avec des styles différents. J'aborde ici des phénomènes qui engagent la figure de l'énonciateur-scripteur numérique: l'énonciation collective, et les styles et lectes multiples qu'un même scripteur peut déployer sur le Web.

\subsubsection{Le scripteur collectif : un singulier pluriel}

En ligne, se manifeste une collectivité assumée par plusieurs scripteurs, qui se remarque à l'œil comme telle. Alors que hors ligne, sur les surfaces imprimées, les écrits produits de manière collaborative sont homogénéisés et linéarisés, en ligne ou sur écran, les différents scripteurs peuvent apparaitre. Le pad, outil d'écriture collaboratif, permet en effet d'écrire à plusieurs dans la matérialité du texte. Etherpad ou Pirate Pad par exemple, éditeurs de texte, mettent en couleur les différents scripteurs. On trouvera l'analyse et le résultat d'une expérience d'écriture à trois scripteurs dans deux billets de carnetières de la plateforme Hypothèses, "Les collect'penseurs - Inscrire, effacer, dialoguer " (Faury 2011) et «[RDV] - Miroir, mon beau miroir...» (Messal 2011). En cliquant sur les images, on peut voir de près ce que donne une écriture à trois, avec l'identification des segments de chacun.e : 


\begin{abstract}
Alors lançons nous : culture matérielle, imaginaire disciplinaire, passage, et, subsidiairement, le thé. et le miroir ! c'est intéressant en lien avec la réflexivité :-pLe miroir c'est une métaphore (?) de la réflexivité. Ah oui, le miroir, toujours. Oui le miroir, le thé, le passage : un syndrome Carollien ? Tant que je ne suis pas le loir endormi, je veux bien!

et si l'on mettait des miroirs au bout de passages ? là où l'on prendrait un thé pour revenir sur nos imaginaires disciplinaires Pas mal! Ça me rappelle un labyrinthe où pour mieux nous perdre, ils positionnaient des miroirs à chaque passage.
\end{abstract}

est-ce que la réflexivité / le miroir fait que l'on se perd ou au contraire que l'on se retrouve?

Commençons : prenez un miroir, que voyez vous dedans ? Le reflet de nos corps mais aussi de nos âmes : la fameuse légende du miroir de vérité.

Ces outils ont en commun de ne pas nécessiter de sauvegarde, ce qui apporte une dimension technoscripturale de plus : le geste de conservation de «l'auteur » est assumé par la machine. Sauf à effacer, le scripteur n'a pas le choix, si l'on peut dire, de la conservation de son texte.

Le cas désormais bien connu du commentaire de blog ou du statut de réseau social constitue également une modalité d'écriture augmentée. La notion d'augmentation, d'abord utilisée pour décrire une intensification des perceptions de la réalité par des systèmes informatiques (dans réalité augmentée), est applicable à l'écriture. Elle permet d'interroger la notion même de textualité en ligne : si l'on veut par exemple reprendre un billet pour le rebloguer, l'intégrer dans un dossier ou un recueil collectif, ou même l'imprimer, que fait-on des commentaires et des réponses ? Les intègre-t-on, tels quels, ou après les avoir triés ? Et sur quels critères? Comment traiter les spams et le trollage? Quelle est donc, finalement, l'unité texte du billet de blog? S'il est repris avec les commentaires, quelle est la valeur de la signature individuelle? C'est par tous ces ricochets la question de l'auctorialité qui se trouve posée, et l'on sait à quel point elle est interrogée et modifiée par les univers discursifs numériques.

\title{
3.3.2. Écritures fluides : la défaite de l'idiolecte
}

51 Dernier phénomène qui affecte la figure du scripteur-énonciateur sur le Web : la fluidité des choix de style et de genre sur le Web. L'idiolecte désigne la langue (au sens très large) d'un individu (ses façons de parler, tournures, usages lexicaux, etc.), et la notion est souvent appliquée aux écrivains : il y aurait un idiolecte balzacien, camusien, proustien, etc. Cela veut dire qu'on reconnaît aux modalités d'écritures d'un auteur des constantes, identifiables par des récurrences. Sur le Web, ce peut être le cas comme ce peut être exactement le contraire : les possibilités d'inscrire son écriture dans des lieux multiples, sous des noms différents, permettent une sorte de polylecte : le scripteur manie plusieurs styles, plusieurs genres de discours, plusieurs répertoires langagiers. Si son identité de chair et d'os est stable (mais qui la connait ?), il exploite au maximum la distinction posée par Ducrot dans les années 1970 : le sujet parlant (l'être dans le monde), est différent de l'énonciateur (celui qui prend la parole, qui inscrit son dire dans une énonciation). Si 
cette distinction n'est pas spécifique car elle est évidemment à l'œuvre dans les univers discursifs hors ligne, elle est particulièrement disponible en ligne: le Web est un dispositif technique qui fournit des affordances pour cela, c'est-à-dire qu'il fournit les possibilités énonciatives: les univers discursifs numériques affordent des possibilités scripturales multiples.

\section{Conclusion}

Les textualités numériques ne sont pas encore saisies par les linguistes TDI comme productions inscrites dans un environnement technodiscursif nécessitant un dispositif d'analyse ad hoc. Trop souvent, les théories qui fonctionnent sur les discours hors ligne sont transportées sur le Web, comme celle de genre ou d'énonciateur. Dans ce travail j'ai essayé de montrer qu'une approche écologique de ces corpus, qui tient étroitement compte des milieux «naturels» des énoncés en ligne, des affordances techniques des environnements, et des remises en jeu de notions aussi fondamentales que l'identité ou l'auctorialité, est nécessaire pour approcher au mieux les traits des discours natifs en ligne.

53 L'alternative entre tradition et innovation, si elle doit être examinée en ce qui concerne les technodiscours, me semble cependant appauvrir les analyses si elle n'est pas accompagnée d'une approche synchronique impliquant une étude de terrain. Si les énoncés hors ligne ne nécessitent pas toujours de compétences techniques spécifiques (comme le précise Vignola, tout le monde «sait lire » un livre), il n'en est pas de même pour les énoncés natifs du Web: comme l'ethnologue doit connaître son terrain de recherche et séjourner chez ses observés, le linguiste analyste des énoncés Web natifs doit détenir une culture numérique, en particulier sous l'angle de la littératie numérique, pour être en mesure de rendre compte de formes langagières composites, imprégnées de technique, qui s'élaborent dans un univers discursif liquide.

\section{BIBLIOGRAPHIE}

Achard-Bayle, Guy et Paveau, Marie-Anne, 2012, « Réel, contexte, et cognition. Contribution à une histoire de la linguistique cognitive », HEL Histoire Épistémologie Langage, vol. 34, n 1 , p. 97-114.

boyd, dana, 2011, « Google +, la dictature des vrais noms », OWNI, [Site], 8 août 2011, http:// owni.fr/2011/08/08/google-plus-dictature-vrais-noms-anonymat-identite/, consulté le 12 janvier 2015.

Brassac, Christian, 2010, « La cognition comme produit de l'interaction sociale. Un point de vue pragmatiste ", Intellectica, $\mathrm{n}^{\circ}$ 53-54, p. 311-330.

Casilli, Antonio, 2010, Les liaisons numériques. Vers une nouvelle sociabilité ?, Paris, Seuil. 
Cislaru, Georgeta, 2009, «Le pseudonyme, nom ou discours ? ", Les Carnets du Cediscor, $\mathrm{n}^{\circ}$ 11, [En ligne], mis en ligne le $1^{\mathrm{er}}$ mars 2011, http://cediscor.revues.org/746, consulté le 12 janvier 2015.

Clark, Alan et Chalmers, David, 1998, « The Extended Mind », Analysis, vol. 58, n 1, p. 10-23.

Culioli, Antoine, 1990, Pour une linguistique de l'énonciation - Opérations et représentations, tome I, Paris-Gap, Ophrys.

Dacos, Marin et Mounier, Pierre, 2010, L'Édition électronique, Paris, La Découverte.

Dervin, Fred et Abbas, Yasmine (dir.), 2009, Technologies numériques du soi et (co-)constructions identitaires, Paris, L'Harmattan.

Faury, Mélodie, 2011, « Les collect'penseurs - Inscrire, effacer, dialoguer », Infuse ! Nos rapports aux sciences, [Carnet de recherche], mis en ligne le 11 octobre 2011, http:// infusoir.hypotheses.org/1521, consulté le 12 janvier 2015.

Foucault, Michel, 1982, Les Techniques de soi, cours à l'université du Vermont, publié dans Technologies of the self, 1988, repris dans Dits et Écrits II, Paris, Gallimard, 2001.

-, 1983, « Usage des plaisirs et techniques de soi », Le Débat, $\mathrm{n}^{\circ} 27$, préface à L'Usage des plaisirs, Gallimard, 1984, repris dans Dits et Écrits II, Gallimard, 2001.

-, 1984, Histoire de la sexualité 3. Le souci de soi, Paris, Gallimard.

Gibson, John, 1979, The Ecological Approach to Visual Perception, Hillsdale, Lawrence Erlbaum.

Hutchins, Edwin, 1995, Cognition in the Wild, Cambridge (MA), Bradford Books-MIT Press.

Lecolle, Michelle et al. (dir.), 2009, Le Nom propre en discours, Les carnets du Cediscor, $\mathrm{n}^{\circ} 11$, [En ligne], http://cediscor.revues.org/729, consulté le 12 janvier 2015.

Laugaa, Maurice, 1986, La pensée du pseudonyme, Paris, PUF.

Lenay, Charles, « Enaction. Externalisme et suppléance perceptive », Intellectica, n 43, 2001, p. 27-52.

Maingueneau, Dominique, 2013, « Genres de discours et web : existe-t-il des genres web ? ", dans Christine Barats (dir.), Manuel d'analyse du web, Paris, Armand Colin, p. 74-93.

Maître Eolas, 2010, « Haut les masques », Journal d'un avocat, [Blog], 30 mai 2010, http:// www.maitre-eolas.fr/post/2010/05/24/Haut-les-masques, consulté le 12 janvier 2015.

Martin, Marcienne, 2006, Le Pseudonyme sur Internet, une nomination située au carrefour de l'anonymat et de la sphère privée, Paris, L'Harmattan.

-, 2012, Se nommer pour exister. L'exemple du pseudonyme sur l'internet, Paris, L'Harmattan.

Mayaffre, Damon, 2005, « De la lexicométrie à la logométrie ", L'Astrolabe. Recherche littéraire et informatique, [En ligne], <hal-00551921>, consulté le 12 janvier 2015.

Messal, Stéphanie, 2011, « [RDV] - Miroir, mon beau miroir... », Misanthropologue, [Carnet de recherche], mis en ligne le 11 octobre 2011, http://misanthropologue.hypotheses.org/251, consulté le 12 janvier 2015.

Norman, Don, 1988, The Design of Everyday Things, New York, Doubleday.

Paveau, Marie-Anne, 2009, " Mais où est donc le sens ? Pour une linguistique symétrique ", dans Pierre Frath et al. (dir.), Actes du colloque international Res per nomen (Reims, 30-31 mai 2009), Reims, Université de Reims, p. 21-31. 
-, 2012a, « Les amitiés numériques. Formes technolangagières et polysémie relationnelle ", conférence au Séminaire PHITECO 2012, Amitié et technologies relationnelles, Université de Technologie de Compiègne, http://penseedudiscours.hypotheses.org/8334, consulté le 12 janvier 2015.

,$- 2012 b$, «Ce que disent les objets. Sens, affordance, cognition », Synergies Pays Riverains de la Baltique, $\mathrm{n}^{\circ}$ 9, p. 53-65 et http://ressources-cla.univ-fcomte.fr/gerflint/Baltique9/baltique9.html, consulté le 12 janvier 2015.

,$- 2012 c$, « Réalité et discursivité. D'autres dimensions pour la théorie du discours ", Semen, nº

34, p. 95-115.

,- 2013a, « Genre de discours et technologie discursive. Tweet, twittécriture et twittérature », Pratiques, $\mathrm{n}^{\circ} 157-158$, p. 7-30.

-, 2013b, « Technodiscursivités natives sur Twitter. Une écologie du discours numérique », Epistémé, $\mathrm{n}^{\circ}$ 9, p. 139-176.

Souchier, Emmanuel et al. (dir.), 2003, Lire,écrire, récrire : objets, signes et pratiques des médias informatisés, Paris, BPI du Centre Pompidou.

Temmar, Malika, 2013, « Qui parle dans les blogs ?», dans Christine Barats (dir.), Manuel d'analyse du web, Paris, Armand Colin, p. 93-97.

Vignola, Éric, 2009, Du blogue au livre. Réflexions sur la nature générique du blogue, Mémoire de maîtrise, Université de Montréal, Département des littératures de langue française, [En ligne], http://fondationlitterairefleurdelys.wordpress.com/2013/04/10/du-blogue-au-livre-reflexionssur-la-nature-generique-du-blogue/, consulté le 12 janvier 2015.

Zythom, 2012, « Tome 2 ", Zythom. Blog d'un informaticien expert judiciaire, mis en ligne le 28 avril 2012, [Blog], http://zythom.blogspot.fr/2012/04/tome-2.html, consulté le 12 janvier 2015.

\section{NOTES}

1. J'emploie l'expression discours natif en ligne pour désigner les discours produits dans les environnements du Web. Elle s'inspire de l'anglais web-native communication et web-native research. 2. J'emploie le terme d'écosystème à la suite de Marin Dacos et Pierre Mounier (2010) qui définissent l'édition électronique comme un écosystème éditorial dans lequel tous les composants sont en relation, formant un ensemble dynamique constamment en mouvement. De même, la plateforme Facebook, comme d'autres réseaux ou lieux numériques, constitue un ensemble de relations dynamiques interdépendantes: la modifcation d'un élément a des répercussions sur l'ensemble du dispositif.

3. Merci à François-Xavier Mas de m'avoir indiqué ces distinctions.

4. J'appelle technosigne un élément iconique non verbal cliquable doté d'une fonction sémanticodiscursive et relationnelle, du type bouton de partage ou like sur Facebook. Le terme et la notion se différencient du «signe passeur» de Souchier et al. (2004), chez lesquels il constitue un hypéronyme de tout segment langagier ou iconique permettant un geste interprétatif de l'usager.

5. Un pad est un éditeur de textes collaboratifs en ligne. Les textes produits font apparaittre les contributions de chaque scripteur via un code couleur (voir fig. 10).

6. Numériqué est un néologisme personnel, et j'utilise aussi numériquer et numérication. 
7. L'étude se situe dans le cadre européen, ou « occidental » selon l'expression consacrée, et un travail sur d'autres aires géographiques et culturelles donnerait sans doute des résultats différents.

8. Au cours d'une discussion dans le séminaire de théorie du discours que j'anime à l'université Paris 13, des étudiants m'ont fait remarquer que le nom de scène, comme le pseudonyme, était choisi et non donné. Le nom de scène ne leur semblait pas vraiment relever du pseudonyme, ou pas de la même manière.

9. L'hétéronymat est le fait de se présenter sous plusieurs noms.

10. dana boyd souhaite que ses nom et prénom soient écrits sans capitales.

\section{RÉSUMÉS}

Le but de cet article est d'identifier et d'analyser les particularités linguistiques de l'écriture en ligne dans ses environnements natifs, pour montrer comment la dimension technique configure les scripturalités. Il voudrait également montrer que, pour étudier les productions langagières en ligne, la connaissance des contextes numériques est nécessaire. En effet, Internet, et le Web en particulier, ne constituent pas de simples supports pour une production scripturale qui s'y transporterait, mais bien des environnements qui configurent structurellement les écritures de manière spécifique. Après l'exposé du cadrage épistémologique et théorique de l'étude, on distingue trois catégories de scripturalité numérique (numérisée, numérique et numériquée), et on interroge la manière dont l'énonciation, au fondement des linguistiques du texte, du discours et de l'interaction, doit être repensée à partir de l'étude des discours en ligne.

The aim of this paper is to identify and analyze the linguistic features of online writing in its native environments, in order to show how the technical dimension configures writing activities. Its purpose is also to show that having a knowledge about digital contexts is necessary to study language productions online. Indeed the Internet and the Web in particular are not mere media for scriptural production, but they are real environments that structurally and specifically configure writings. After the presentation of the epistemological and theoretical framework of the study, we propose to distinguish three categories of digital scripturality (digitized, digital and digitalized), and to ask how enunciation, as a base for text linguistics, discourse analysis and interactionism, needs to be rethought from the study of online discourse.

\section{INDEX}

Mots-clés : analyse du discours numérique, délinéarisation, discours natif en ligne, postdualisme, pseudonymat, technodiscours

Keywords : digital discourse analysis, delinearization, webnative discourse, pseudonymity, technodiscourse 


\section{AUTEUR}

\section{MARIE-ANNE PAVEAU}

Université Paris 13, Sorbonne Paris Cité, Pléiade (EA 7338) 